\title{
Escherichia Coli B121: A Useful Biocatalyst for the Synthesis of Purine Nucleosides
}

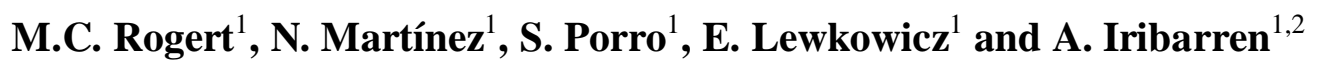 \\ ${ }^{1}$ Universidad Nacional de Quilmes. R. S. Peña 180, (1876) Bernal, Buenos Aires, Argentina \\ ${ }^{2}$ INGEBI, CONICET, Vuelta de Obligado 2490, (1428) Buenos Aires, Argentina \\ E-mail: elewko@unq.edu.ar
}

\begin{abstract}
E. coli BL21 cells were able to synthesize several purine nucleosides from pyrimidine ones. Kinetics and yields of this reaction showed a strong dependence on $\mathrm{pH}$, temperature, reagent concentrations and weight of wet cell paste. Yields over $90 \%$ were reached in the synthesis of adenosine.
\end{abstract}

\section{Introduction}

Modified nucleosides are wide diffused as antiviral and antitumor drugs as well as starting materials for antisense oligonucleotides. In contrast to classical chemical synthesis of these, good results as far as yield, simplicity and regiostereospecificity are concerned can be achieved at low cost using pure enzymes or whole cells as biocatalysts.

The methodology employed in the synthesis of purine nucleosides involves the transfer of a sugar residue from a donor pyrimidine nucleoside to an acceptor purine base. This process requires the presence of enzymes belonging to the family of transferases, specially the purine and pyrimidine nucleoside phosphorylases (PNP and PyNP) which are present in most of the microorganisms [1].

In this work, several experimental variables have been studied in order to select the best conditions necessary for the E.Coli BL21 catalyzed synthesis of purine nucleosides.

\section{Experimental}

The E. coli strain was grown in Lb medium in shaked flasks at $27^{\circ} \mathrm{C}$ until saturation, centrifuged and washed with the suitable buffer. The resultant wet cell paste was used as catalyst of the reaction [2] after suspending in phosphate buffer $(5 \mathrm{ml})$ and addition of the nucleoside and purine base. The mixture was stirred and heated at constant temperature in glycerin bath and the reaction products were analyzed by tlc and hplc. 


\section{Results and Discussion}

One of the biotransformations studied in this work is shown in Figure 1.

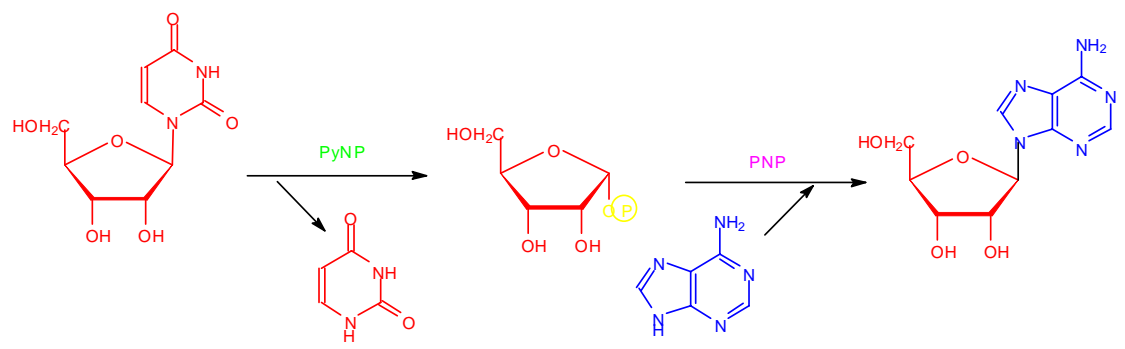

Figure 1. Enzymatic synthesis of adenosine.

The reagents tested were the nucleosides uridine and thymidine and the bases adenine, hypoxanthine and guanine. The following variables have been studied: temperature, reagent concentrations, $\mathrm{pH}$ and buffer concentration, biocatalyst amount and reaction time [3]. For example, adenosine was obtained with a yield higher than $90 \%$ working at $60^{\circ} \mathrm{C}$ in $30 \mathrm{mM}$ phosphate buffer at $\mathrm{pH} 7$, with excess of uridine during 1 hour. Longer reaction times have been observed when tymidine was used as ribose donor.

\section{References and Notes}

1. Pal, S.; Nair, V. Biocatal. and Biotransf. 1997, 15, 147.

2. Utagawa, T.; Morisawa, H.; Yoshinaga, F.; Yamazaki, A.; Mitsugi, K.; Hirose, Y. Agric. Biol.Chem. 1985, 49 (4), 1053.

3. Krenitsky, T.; Koszalka, G.; Tuttle, J. Biochemistry 1981, 20, 3615. 